\title{
Biliary metal stents are superior to plastic stents for preoperative biliary decompression in pancreatic cancer
}

\author{
Christopher Decker · John D. Christein • \\ Milind A. Phadnis • C. Mel Wilcox • \\ Shyam Varadarajulu
}

Received: 26 May 2010/ Accepted: 24 November 2010/Published online: 4 March 2011

(c) The Author(s) 2011. This article is published with open access at Springerlink.com

\begin{abstract}
Background It is unclear whether plastic or metal stents are more suitable for preoperative biliary decompression in pancreatic cancer. The objective of this study was to compare the rate of endoscopic reinterventions in patients with pancreatic cancer undergoing plastic or self-expandable metal stent (SEMS) placements for preoperative biliary decompression. Methods This was a retrospective study of all patients with obstructive jaundice secondary to pancreatic head cancer who underwent their index endoscopic retrograde cholangiopancreatography (ERCP) and all follow-up biliary stent placements at our center before undergoing pancreaticoduodenectomy. Plastic or SEMS were placed at ERCP for biliary decompression. The main outcome measure was to compare the rate of endoscopic reinterventions between the plastic and SEMS cohorts.

Results 29 patients who underwent pancreaticoduodenectomy had preoperative biliary stent placement (18 plastic, 11 SEMS) at our center. Whereas none of the 11 patients who
\end{abstract}

Electronic supplementary material The online version of this article (doi:10.1007/s00464-010-1552-6) contains supplementary material, which is available to authorized users.

C. Decker - M. A. Phadnis - C. Mel Wilcox · S. Varadarajulu Division of Gastroenterology-Hepatology,

University of Alabama at Birmingham School of Medicine,

Birmingham, AL, USA

J. D. Christein

Department of Surgery, University of Alabama

at Birmingham School of Medicine, Birmingham, AL, USA

S. Varadarajulu $(\square)$

Basil Hirschowitz Endoscopic Center of Excellence,

University of Alabama at Birmingham School of Medicine,

Birmingham, AL 35294-0007, USA

e-mail: svaradarajulu@yahoo.com underwent SEMS placement had stent dysfunction, 7 of 18 (39\%) patients with plastic stents required endoscopic reintervention before surgery $(P=0.02)$. Reinterventions were due to cholangitis $(n=1)$ or persistent elevation in serum bilirubin levels $(n=6)$. Two patients with SEMS underwent EUS-guided fine-needle aspiration after ERCP, which yielded a positive diagnosis of cancer in all cases; SEMS did not impair visualization of the tumor mass at EUS. Pancreaticoduodenectomy was undertaken successfully in all 29 patients and the presence of a SEMS did not interfere with biliary anastomosis. On univariate logistic regression, only SEMS placement was associated with less need for endoscopic reintervention $(P=0.02)$.

Conclusions SEMS are superior to plastic stents for preoperative biliary decompression in pancreatic cancer.

Keywords Metal stents - Plastic stents - Preoperative · Biliary decompression - Pancreatic cancer .

Pancreaticoduodenectomy

Pancreatic cancer is the fourth leading cause of cancerrelated deaths in the United States. Cancer of the head of the pancreas causes biliary obstruction with curative option being pancreaticoduodenectomy (Whipple's procedure). Preoperative endoscopic retrograde cholangiopancreatography (ERCP) is usually undertaken in patients with resectable disease to relieve cholestasis from biliary obstruction, which is thought to impair immune response, clotting, and other functions that impact intraoperative and postoperative outcomes [1-3]. Despite conflicting data pertaining to preoperative biliary drainage, ERCP with biliary stenting has become standard practice in patients with pancreatic head cancer [4-6]. In a recent, multicenter, randomized trial, patients who underwent preoperative 
biliary drainage had a $74 \%$ rate of complications compared with $39 \%$ for those who directly underwent surgery [7]. All patients in this trial underwent placement of plastic stents. In ERCP, self-expandable metal stent (SEMS) are being increasingly placed for palliation of malignant obstructive jaundice. Compared with plastic stents, SEMS are of large caliber and have demonstrated longer patency in randomized trials [8]. Even in patients with resectable disease, short SEMS when placed below the level of transection do not impair technical outcomes at surgery and can be safely removed along with the surgical specimen [9].

This retrospective study was designed to compare the rates of endoscopic reintervention in patients with pancreatic cancer who underwent SEMS or plastic stent placement for preoperative biliary decompression.

\section{Materials and methods}

The surgical and ERCP databases were queried for all patients with pancreatic cancer who underwent preoperative endoscopic biliary decompression at the University of Alabama at Birmingham from January 2006 to December 2009. The ERCP database comprises 72 prospectively collected variables, which include patient demographics, laboratory indices and investigations, previous endoscopic interventions, procedural details, and complications. The surgical database comprises technical outcomes and complications pertaining to pancreaticoduodenectomy.

Included in the study were all patients (aged $>19$ year) with pancreatic head cancer who underwent their index ERCP and all follow-up biliary stent placements at our center before undergoing pancreaticoduodenectomy. Excluded were patients with distant metastasis who underwent palliative surgery, biliary intervention (percutaneous biliary drainage catheter or ERCP) performed at outside hospitals for presenting complaints, tumor-related gastric outlet obstruction, cholangitis at clinical presentation, and those with postsurgical anatomy.

All ERCPs were performed by two attending endoscopists (CMW, SV) under conscious sedation using a combination of intravenous midazolam, meperidine, and ketamine. At ERCP, plastic (10-Fr; length 7-9 cm; Wilson-Cook Medical Corporation, Winston-Salem, NC) or SEMS (10 mm; length, $6 \mathrm{~cm}$; Boston Scientific Corporation, Nattick, MA) were placed for biliary decompression. None of the study patients received prophylactic antibiotics or required reversal medications for coagulation disorders.

\section{Statistical analysis}

Patients who underwent plastic and SEMS were compared for their serum bilirubin levels, tumor size, stricture length, time between stent placement and surgery, and length of stay in the hospital after surgery. Continuous variables have been reported as means (standard deviations) and medians (IQR) and compared by a two-sample $t$ test or the Wilcoxon rank-sum test. Categorical variables have been reported in terms of their frequencies and the two proportions were compared by using the Fisher's exact test.

A Fisher's exact test was performed to determine whether having an endoscopic reintervention is associated with the type of stent deployed. Similarly, a Fisher's exact test was deemed appropriate for assessing whether having an endoscopic reintervention was associated with the categorical predictors of interest. Univariate logistic regression was performed to assess whether the other predictors (continuous variables) of interest increased or decreased the odds of having an endoscopic reintervention. Crude odds ratios and their corresponding $95 \%$ confidence intervals (CIs) were calculated for the same. The SAS statistical software was used for these analyses, and the $P$ values were reported for a two-sided test. Type I error was set at the 0.05 level of significance.

\section{Evaluation of outcomes}

The primary outcome was to compare the rate of endoscopic reintervention between patients who underwent plastic or SEMS placement for preoperative biliary decompression. Reintervention was defined as the need for stent exchange when patients developed postprocedural cholangitis or had persistent hyperbilirubinemia $(<50 \%$ decline in serum bilirubin levels after initial stent placement).

\section{Results}

29 (men 55\%, Caucasians 59\%) of 270 patients (11\%) who underwent pancreaticoduodenectomy had their index ERCP performed at our institution and constitute the study cohort. 98 patients who had biliary interventions performed at outside hospitals and underwent pancreaticoduodenectomy at our institution were excluded. 18 patients $(62 \%)$ underwent plastic and $11(38 \%)$ underwent SEMS placements. There was no difference in patient demographics between both cohorts. The mean age of the patients was 55 year [standard deviation $(\mathrm{SD})=10.8$ ] and 62 year $(\mathrm{SD}=14.2)$ between patients who underwent metal and plastic stents, respectively. Four of $11(37 \%)$ and 9 of 18 $(50 \%)$ patients who underwent metal and plastic stent placements, respectively, were women. Except for postsurgical length of stay in the hospital, there was no difference in serum bilirubin levels, size of the pancreatic 
mass (largest dimension on computed tomogram [CT]), length of biliary stricture at ERCP, and median duration between stent placement until surgery between patients who underwent plastic or SEMS placements (Table 1). Both endoscopists had a lifetime experience of more than 3,000 ERCP procedures and performed 58\% (SV) and $42 \%$ (CMW) of all subjects recruited to this study without difference between both cohorts.

Whereas all 11 patients who underwent SEMS placement had successful resolution of jaundice and did not require reintervention, 7 of 18 patients (39\%) who underwent plastic stent placement underwent a repeat ERCP for stent exchange. One of seven patients developed cholangitis and six had persistent elevation in serum bilirubin levels. The patient who developed cholangitis required hospitalization for 4 days. Three other patients with persistent hyperbilirubinemia had severe pruritus, which required inpatient hospitalization (median stay, 2 days) for repeat ERCP.

At repeat ERCP, the plastic stents were replaced successfully (with 10-Fr plastic stents) and the bilirubin levels declined in all patients. No patient in either cohort developed other ERCP-related complications, such as pancreatitis or perforation. After stenting, two patients in the SEMS cohort and one in the plastic stent cohort underwent EUSguided fine-needle aspiration (FNA), which was diagnostic of cancer in all three patients. The presence of a SEMS did not impact visualization or tissue yield at EUS-guided FNA. During the study period, none of the patients who had their index ERCP and subsequent pancreaticoduodenectomy at our institution had any other biliary interventions performed at outside hospitals.

At surgery, there was no technical difficulty in removing the SEMS in any patient. The fully covered metal stent could be removed without any resistance due to the presence of a smooth coating, and the partially covered metal stent required minimal force to be retrieved from the bile duct (Video 1). Also, there was no difference in the rate of postoperative complications between patients who underwent SEMS or plastic stent placements. Two of $11(18 \%)$ patients who underwent SEMS placement developed postsurgical complications (surgical wound infection managed conservatively in 1 and required debridement in the other) compared with 4 of 18 (22\%) patients (postsurgical bleeding in 2, which was managed conservatively in 1 and resulted in death in a Jehovah's witness who refused transfusion, wound infection managed by debridement in 1 , and death due to cardiopulmonary arrest in another) who underwent plastic stent placement $(P=1.0)$.

When factors associated with endoscopic reintervention were analyzed (Table 2), plastic stent placement was associated with significant increase in the rates of endoscopic reintervention $(P=0.026)$. None of the other variables were found to increase or decrease significantly the odds of having a reintervention and hence a multivariable analysis was not performed. Also, because none of the patients who underwent SEMS placements required reintervention, it was not possible to compute crude or adjusted odds ratios.

Table 1 Comparison of the clinical characteristics of the plastic and metal stent cohorts

\begin{tabular}{llll}
\hline Variable & Plastic stent $(n=18)$ & Metal stent $(n=11)$ & $P$ value \\
\hline Median (IQR) time between stenting and surgery (days) & $24(10-30)$ & $17(12-33)$ & $11(8.77)$ \\
Mean (SD) serum bilirubin levels (mg/dl) & $12.28(6.36)$ & $24.73(6.68)$ \\
Mean (SD) tumor size on CT (mm) & $29(8.31)$ & $30.91(5.82)$ & $0.655^{* *}$ \\
Mean (SD) stricture length & $31.61(11.45)$ & $7(7-8)$ & $0.161^{* *}$ \\
Median (IQR) length of stay after surgery (days) & $9(8-12)$ & $0.829^{* *}$ \\
\hline
\end{tabular}

* Unpaired two sample $t$ test; ** Wilcoxon rank-sum test (SD)

$S D$ standard deviation; $I Q R$ interquartile range

Table 2 Univariate analysis for evaluating factors associated with endoscopic reintervention

* $P$ values calculated using Fisher's exact test; $C I$ confidence interval

\begin{tabular}{llll}
\hline Variable & Odds ratio & $95 \%$ CI & $P$ value \\
\hline Time between stent placement and surgery & 1 & $(0.991,1.011)$ & 0.83 \\
Length of stay after surgery & 1.14 & $(0.868,1.485)$ & 0.353 \\
Serum bilirubin levels & 1.01 & $(0.892,1.133)$ & 0.925 \\
Tumor size on CT & 1.05 & $(0.941,1.177)$ & 0.367 \\
Stricture length at ERCP & 1.08 & $(0.973,1.19)$ & 0.152 \\
Postoperative complications & 0.77 & $(0.11,5.16)$ & 1 \\
Stent type (plastic vs. metal) & - & - & $0.026^{*}$ \\
\hline
\end{tabular}




\section{Discussion}

In this study, patients who underwent SEMS placements required no endoscopic reintervention before undergoing pancreaticoduodenectomy. On the contrary, nearly $40 \%$ of patients who underwent plastic stent placement required a stent exchange due to development of cholangitis or persistent hyperbilirubinemia.

Plastic biliary stents occlude at a considerably higher rate and earlier than SEMS, which have a diameter that is 3 times that of plastic stents. Therefore, most major pancreaticobiliary centers no longer place plastic stents for good functional patients with inoperable disease. Of late, a new strategy is evolving where short-length $(4-6 \mathrm{~cm})$ SEMS are being placed in patients who undergo preoperative ERCP [10]. If resectable disease is confirmed at surgery, the stent is removed with the surgical specimen [9]. If the disease is deemed inoperable at surgery, the patient's jaundice being already palliated does not require a major biliary diversion surgery $[9,10]$. Also, fully covered and removable SEMS are currently available and their use may have additional implications for patients with resectable pancreatic cancer [11]. Although the impact of SEMS on tissue procurement is unknown, in this study, the presence of a metal stent in the bile duct did not obscure tumor imaging or diminish tissue yield at EUS. Although we observed no difference in the rates of postoperative complications between both cohorts, a small sample size could have precluded meaningful assessment of this outcome.

In a recent randomized trial that compared preoperative biliary drainage with surgery alone for patients with pancreatic cancer, cholangitis related to stent dysfunction developed in $26 \%$ of patients and another one-third of patients underwent palliative biliary bypass surgery [7]. Also, 30\% of patients required a stent exchange before surgery. These stent-related complications and the need for endoscopic reintervention could have been potentially avoided if SEMS were deployed instead of plastic stents. Also, preoperative adjuvant chemoradiation is being administered more often to patients with cancer of the pancreatic head, thereby further delaying surgery [12]. SEMS have been shown to provide adequate and durable biliary decompression in these patients [13].

Limitations of this study include its retrospective design and the small numbers of enrolled subjects. Nevertheless, the findings are significant given the careful selection of subjects who did not have prior endoscopic interventions and all procedures were performed at one center by dedicated endoscopists and one surgeon. Randomized trials are in progress to compare the plastic and SEMS for preoperative biliary decompression in pancreatic cancer.

Disclosures Dr. Christopher Decker, Dr. John Christein, Dr. Milind Phadnis, and Dr. Mel Wilcox have no conflicts of interest or financial ties to disclose. Dr. Shyam Varadarajulu is a consultant for Boston Scientific Corporation and Olympus Medical Systems Corporation, Tokyo.
Open Access This article is distributed under the terms of the Creative Commons Attribution Noncommercial License which permits any noncommercial use, distribution, and reproduction in any medium, provided the original author(s) and source are credited.

\section{References}

1. Van der Gaag NA, Kloek JJ, de Castro SM, Busch OR, van Gulik TM, Gouma DJ (2009) Preoperative biliary drainage in patients with obstructive jaundice: history and current status. J Gastrointest Surg 13:814-820

2. Klinkenbijl JH, Jeekel J, Schmitz PI, Rombout PA, Nix GA, Bruining HA, van Blankenstein M (1993) Carcinoma of the pancreas and periampullary region: palliation versus cure. $\mathrm{Br} \mathrm{J}$ Surg 80:1575-1578

3. Trede M, Schwall G (1988) The complications of pancreatectomy. Ann Surg 207:39-47

4. Lillemoe KD (1999) Preoperative biliary drainage and surgical outcome. Ann Surg 230:143-144

5. Sewnath ME, Birjmohun RC, Rauws EA, Huibregste K, Obertop H, Gouma DJ (2001) The effect of preoperative biliary drainage on postoperative complications after pancreaticoduodenectomy. J Am Coll Surg 192:726-734

6. Pisters PW, Hudec WA, Hess KR, Lee JE, Vauthey JN, Lahoti S, Raijman I, Evans DB (2001) Effect of preoperative biliary decompression on pancreaticoduodenectomy-associated morbidity in 300 consecutive patients. Ann Surg 234:47-55

7. van der Gaag NA, Rauws EA, van Eijck CH, Bruno MJ, van der Harst E, Kubben FJ, Gerritsen JJ, Greve JW, Gerhards MF, de Hingh IH, Klinkenbijl JH, Nio CY, de Castro SM, Busch OR, van Gulik TM, Bossuyt PM, Gouma DJ (2010) Preoperative biliary drainage for cancer of the head of the pancreas. N Engl J Med 362:129-137

8. Moss AC, Morris E, MacMathuna P (2006) Palliative biliary stents for obstructing pancreatic carcinoma. Cochrane Database Syst Rev 1:CD004200

9. Lawrence C, Howell DA, Conklin DE, Stefan AM, Martin RF (2006) Delayed pancreaticoduodenectomy for cancer patients with prior ERCP-placed, nonforeshortening, self-expanding metal stents: a positive outcome. Gastrointest Endosc 63:804-807

10. Chen VK, Arguedas MR, Baron TH (2005) Expandable metal biliary stents before pancreaticoduodenectomy for pancreatic cancer: a Monte-Carlo decision analysis. Clin Gastroenterol Hepatol 3:1229-1237

11. Kahaleh M, Brock A, Conawar MR, Shami VM, Dumonceau JM, Northup PG, Tokar J, Rich TA, Adams RB, Yeaton P (2007) Covered self-expandable metal stents in pancreatic malignancy regardless of resectability: a new concept validated by a decision analysis. Endoscopy 39:319-324

12. Landry J, Catalano PJ, Staley C, Harris W, Hoffman J, Talamonti M, Xu N, Cooper H, Benson AB 3rd (2010) Randomized phase II study of gemcitabine plus radiotherapy versus gemcitabine, 5-fluorouracil, and cisplatin followed by radiotherapy and 5-fluorouracil for patients with locally advanced, potentially resectable pancreatic adenocarcinoma. J Surg Oncol 101:587-592

13. Varadhachary GR, Wolff RA, Crane $\mathrm{CH}$, Sun CC, Lee JE, Pisters PW, Vauthey JN, Abdalla E, Wang H, Staerkel GA, Lee JH, Ross WA, Tamm EP, Bhosale PR, Krishnan S, Das P, Ho L, Xiong H, Abbruzzese JL, Evans DB (2008) Preoperative gemcitabine and cisplatin followed by gemcitabine-based chemoradiation for resectable adenocarcinoma of the pancreatic head. J Clin Oncol 26:3487-3495 